\title{
Molecular mechanisms underlying ochratoxin A-induced genotoxicity: global gene expression analysis suggests induction of DNA double-strand breaks and cell cycle progression
}

\author{
Daisuke Hibi', Aki Kijima', Ken Kuroda', Yuta Suzuki', Yuji Ishii', Meilan Jin', \\ Masahiro Nakajima ${ }^{2}$, Yoshiko Sugita-Konishi ${ }^{3}$, Tokuma Yanai ${ }^{4}$, Takehiko Nohmi ${ }^{5}$, \\ Akiyoshi Nishikawa ${ }^{6}$ and Takashi Umemura ${ }^{1}$ \\ ${ }^{1}$ Division of Pathology, National Institute of Health Sciences, 1-18-1 Kamiyoga, Setagaya-ku, Tokyo 158-8501, Japan \\ ${ }^{2}$ Environmental Health Department, Nagoya City Public Health Institute, \\ 1-11, Hagiyama-cho, Mizuho-ku, Nagoya 46-8615, Japan \\ ${ }^{3}$ Division of Microbiology, National Institute of Health Sciences, 1-18-1 Kamiyoga, Setagaya-ku, Tokyo 158-8501, Japan \\ ${ }^{4}$ Laboratory of Veterinary Pathology, Department of Veterinary Medicine, Faculty of Applied Biological Sciences, \\ Gifu University, 1-1 Yanagido, Gifu 501-1193, Japan \\ 5Division of Genetics and Mutagenesis, National Institute of Health Sciences, \\ 1-18-1 Kamiyoga, Setagaya-ku, Tokyo 158-8501, Japan \\ ${ }^{6}$ Biological Safety Research Center, National Institute of Health Sciences, \\ 1-18-1 Kamiyoga, Setagaya-ku, Tokyo 158-8501, Japan
}

(Received November 1, 2012; Accepted November 26, 2012)

\begin{abstract}
Ochratoxin A (OTA) is a renal carcinogen primarily affecting the S3 segment of proximal tubules in rodents. In our previous study, we reported that OTA induces reporter gene mutations, primarily deletion mutations, in the renal outer medulla (OM), specifically in the S3 segment. In the present study, to identify genes involved in OTA-induced genotoxicity, we conducted a comparative analysis of global gene expression in the renal cortex (COR) and OM of kidneys from gpt delta rats administered OTA at a carcinogenic dose for 4 weeks. Genes associated with DNA damage and DNA damage repair, and cell cycle regulation were site-specifically changed in the OM. Interestingly, genes that were deregulated in the OM possessed molecular functions such as DNA double-strand break (DSB) repair (Rad18, Brip1, and Brcc3), cell cycle progression (Cyce1, Ccna2, and Ccnb1), $\mathrm{G}_{2} / \mathrm{M}$ arrest in response to DNA damage (Chek1 and Wee1), and p53-associated factors (Phlda3 and Ccng1). Significant increases in the mRNA levels of many of these genes were observed in the OM using real-time RT-PCR. However, genes related to oxidative stress exhibited no differences in either the number or function of altered genes in both the OM and COR. These results suggested that OTA induced DSB and cell cycle progression at the target site. These events other than oxidative stress could trigger genotoxicity leading to OTA-induced renal tumorigenicity.
\end{abstract}

Key words: Apoptosis, DNA damage, Karyomegaly, Microarray, Mycotoxin

\section{INTRODUCTION}

Ochratoxin A (OTA) is a mycotoxin produced by fungal species and is often found in food as a contaminant (EFSA, 2006). OTA is a well-known nephrotoxicant and renal carcinogen in rodents (NTP, 1989) and may be responsible for Balkan endemic nephropathy and urinary tract tumors in humans (JECFA, 2001). Humans are still exposed to OTA in various foods (EFSA, 2006), and currently, there is no way to prevent exposure to this contaminant. However, the mechanism underlying OTA-induced renal tumorigenesis, especially the possible participation of genotoxic mechanisms, has been the subject of controversy since conventional in vitro and in vivo mutagenicity

Correspondence: Takashi Umemura (E-mail: umemura@nihs.go.jp) 


\section{Hibi et al.}

tests for OTA have not demonstrated consistent outcomes (JECFA, 2008).

It has been suggested that OTA behaves according to characteristic kinetics in the kidneys, possibly due to the existence of specific transporters which cause localization toxicity at the S3 segment in the renal proximal tubules (Pfohl-Leszkowicz and Manderville, 2007; Dahlmann et al., 1998). In addition, OTA has been also reported to cause renal tumors at the same site (NTP, 1989; Bendele et al., 1985; Boorman et al., 1992). In our previous study, we observed nonuniform distribution of OTA in the kidney and therefore conducted site-specific in vivo mutation analysis using gpt delta rats, in which reporter gene mutation assays were performed separately in the renal cortex (COR) and outer medulla (OM). As a result, significant increases in the mutant frequency (MF) of the $\mathrm{red} / \mathrm{gam}$ gene (Spi-) were observed in the OM (primarily in the S3 segment), but not in the COR, suggesting the involvement of genotoxic mechanisms in OTA-induced renal carcinogenesis (Hibi et al., 2011).

Based on the positive Spi- assay (Nohmi et al., 2000, 2005 ), it is possible that OTA can induce deletion mutations at the carcinogenic target site. In addition to the fact that OTA-modified bases lead to the formation of apurinic sites (Cavin et al., 2007), the positive outcome in the alkaline comet assay allowed us to speculate that OTA induces DNA strand breaks followed by deletion mutations (Zeljezić et al., 2006). Otherwise, although there were no changes in 8-hydroxydeoxyguanine (8-OHdG) levels in kidney DNA extracted from the OM in the above-mentioned study, several lines of evidence, such as the generation of reactive oxygen species (ROS) (Schaaf et al., 2002), the inhibition of Nrf-2 activity (Cavin et al., 2007), and the positive results in formamidopyrimidineDNA glycosylase (Fpg)-modified comet assays (Kamp et al., 2005), indicate the possibility that oxidative DNA damage triggers these mutations. In any event, the precise mechanisms facilitating OTA-induced gene mutations in vivo remain uncertain (JECFA, 2008). Microarray analysis of global gene expression is a powerful tool to identify candidate genes associated with various pathogenic conditions. Marin-Kuan et al. (2006) reported the comprehensive analysis of gene expression in the kidneys and liver of rats treated with OTA, demonstrating the alteration of cellular homeostasis, including inhibition of anti-oxidative defense. However, it is highly probable that sitespecific analysis of gene alterations may be necessary to clarify the molecular processes involved in OTA-induced DNA damage and consequent gene mutations due to the characteristic distribution of OTA in the kidneys.

In the present study, to investigate the detailed mech- anisms involved in OTA-induced carcinogenesis, a comparison of changes in gene expression between the COR and OM was performed in the kidneys of rats treated with OTA at a carcinogenic dose for 4 weeks. In particular, we focused on the genes associated with DNA damage and DNA damage repair, oxidative stress, the cell cycle, and apoptosis, and some of these changes were also confirmed by real-time RT-PCR methods.

\section{MATERIALS AND METHODS}

\section{Experimental animals and housing conditions}

The protocol for this study was approved by the Animal Care and Utilization Committee of the National Institute of Health Sciences. Specific pathogen-free, five-weekold male F344/NSlc-Tg ( $g p t$ delta) rats carrying about 5 tandem copies of the transgene lambda EG10 per haploid genome were obtained from Japan SLC (Shizuoka, Japan) and acclimated for 1 week prior to testing. Animals were housed in a room with a barrier system and maintained under constant temperature $\left(23 \pm 2{ }^{\circ} \mathrm{C}\right)$, relative humidity $(55 \pm 5 \%)$, air change (12 times/hr), and lighting (12-hr light-dark cycle), with free access to an Oriental CRF-1 basal diet (Oriental Yeast Co., Ltd., Tokyo, Japan) and tap water.

\section{Test compound}

OTA was extracted from a culture of Aspergillus ochraceus (BD-5). Fermentative production of OTA was performed based on the culture and extraction methods of Kumata et al. (1980), with minor modifications. Several 500-ml Erlenmeyer flasks containing $100 \mathrm{~g}$ polished rice were autoclaved before the addition of $20 \mathrm{ml}$ sterilized water. The rice was inoculated with $A$. ochraceus spores and incubated for 2 weeks at $25^{\circ} \mathrm{C}$. After incubation, $200 \mathrm{ml}$ of a chloroform-acetic acid mixture (99:1) was added to the moldy rice in each flask. The extracts collected from the flasks were concentrated and precipitated with 41 hexane on a stirrer. The precipitate was dissolved in $500 \mathrm{ml}$ chloroform and subjected to chromatography using a silica gel column and mobile phases of benzene-acetic acid (100:0 to $88: 12$ ) with a linear gradient. The benzene-acetic acid (95:5 and 92.5:7.5) eluates were evaporated to dryness. Benzene was added to the extract, and the solution was heated, followed by gentle cooling, for crystallization of OTA. The purification of crystals was confirmed by high-performance liquid chromatography (HPLC). The purity of OTA was estimated to be greater than $95 \%$ from the area percentage of the chromatogram (data not shown). 
Molecular mechanisms underlying OTA-induced genotoxicity

\section{Animal treatment}

Groups of 5 male gpt delta rats were fed 5 ppm OTA or a basal diet without supplement for 4 weeks. The dietary dose level of $5 \mathrm{ppm}$ was selected based on the carcinogenic dose reported in a 2-year carcinogenicity study of OTA in rats (Mantle et al., 2005). At necropsy, as previously described, the harvested kidneys were divided into the cortex (COR), outer medulla (OM), and inner medulla (Hibi et al., 2011). The CORs and OMs were stored at $-80^{\circ} \mathrm{C}$ for cDNA microarray and real-time RT-PCR.

\section{RNA isolation}

The CORs and OMs from all animals were soaked overnight in RNAlater-ICE (Applied Biosystems/Ambion, Austin, TX, USA) at $-20^{\circ} \mathrm{C}$, and then total RNA was isolated using QIAGEN RNeasy Mini Kits (QIAGEN $\mathrm{GmbH}$, Hilden, Germany) following the standard Mini Protocol for animal tissues. Briefly, COR and OM sections (15-20 mg each) were placed in $600 \mu \mathrm{l}$ of QIAGEN RLT buffer and homogenized. Homogenates were centrifuged to remove particulate debris, and debris-free lysates were mixed with equal volumes of $70 \%$ ethanol and loaded on RNeasy spin columns. The samples were then washed 3 times on the column, and RNA was extracted in $30 \mu \mathrm{l}$ RNase-free water. Spectrophotometric determination of the absorbance at 260 and $280 \mathrm{~nm}$ was made, and these measurements were used to determine the purity, concentration (ng/ $\mu \mathrm{l})$, and total yield (ng) of RNA samples. In addition, assessment of RNA quality and measurement of the $28 \mathrm{~S}$ to $18 \mathrm{~S}$ ratio was performed using an Agilent 2100 Bioanalyzer (Agilent Technologies, Santa Clara, CA, USA).

\section{cDNA microarray}

In the 3 male rats in the group, purified total RNA (100 ng) from each site was labeled with Cyanine-3 dye using the Low Input Quick Amp Labeling Kit (Agilent Technologies). RNA concentration and dye incorporation were measured using a UV-VIS spectrophotometer (Nanodrop ND-1000, NanoDrop Technologies, Wilmington, DE, USA). RNA quality was assessed using an Agilent 2100 Bioanalyzer (Agilent Technologies). Fluorescently labeled cRNA was hybridized to $4 \times 44 \mathrm{~K}$ whole rat genome microarray gene expression chips (Agilent Technologies). Hybridization and wash processes were performed following the manufacturer's protocol (Agilent Technologies), and hybridized microarray chips were scanned using an Agilent Microarray Scanner (Model G2565BA, Agilent Technologies). Feature Extraction software (Agilent Technologies) was employed for the imaging analysis and data extraction processes. Nor- malization of gene expression data and filtering probe sets by expression levels, flags, and errors were performed. Differences in COR and OM gene expression between treated and control rats were analyzed by analysis of variance (t-test, cut-off value: $P<0.05$, multiple testing correction: Benjamini hochburg FDR). For the complete data at each site, a gene ontology approach was used, and DNA damage and DNA damage repair, oxidative stress, the cell cycle, apoptosis, and related genes were extracted.

\section{Quantitative real-time RT-PCR for mRNA expression}

Several key genes, each of which represent a category of genes found to be modulated by OTA, were selected to validate the microarray data. In all animals, cDNA copies of total RNA from each site were obtained using a High Capacity cDNA Reverse Transcription kit (Applied Biosystems Japan Ltd.). All PCR reactions were performed with primers for rat representative genes in the microarray data and TaqMan Rodent GAPDH Control Reagents as an endogenous reference in the Applied Biosystems 7900HT FAST Real-Time PCR Systems using the TaqMan Fast Universal PCR Master Mix and TaqMan Gene Expression Assays (Applied Biosystems Japan Ltd.). Expression levels of target genes were calculated by the relative standard curve method and were determined as ratios to GAPDH levels. Data were presented as fold-change values of OTA-treated samples relative to controls at each site. Data were analyzed statistically as follows. Variance in the data was checked for homogeneity with the F-test. When the data were homogeneous, a Student's t-test was applied. In heterogeneous cases, Welch's t-test was used instead.

\section{RESULTS}

\section{Global gene expression analysis in the COR and OM}

Under the cut-off condition of $P<0.05$ and at least 1.5 -fold change values, the expression of 1,796 genes in the COR (upregulation: 523, downregulation: 1,273) and 1726 genes in the OM (upregulation: 716, downregulation: 1010) were differentially modulated by OTA treatment. A total of 999 genes were deregulated in the COR only (upregulation: 346, downregulation: 653), while 929 genes were deregulated in the OM only (upregulation: 537, downregulation: 392). A total of 797 common genes were altered in both sites. For the genes that were up- or downregulated by OTA, a gene ontology approach was conducted, and DNA damage-, DNA damage repair-, oxidative stress-, cell cycle-, and apoptosis-related genes 
a)

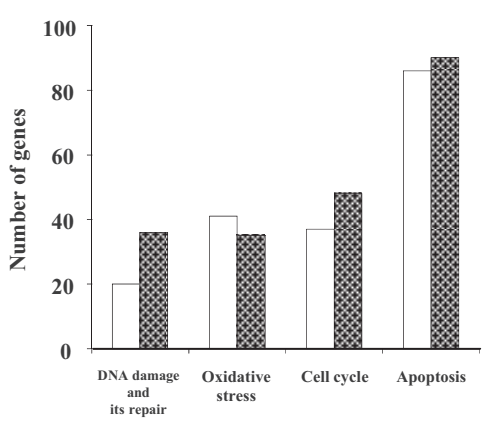

d)

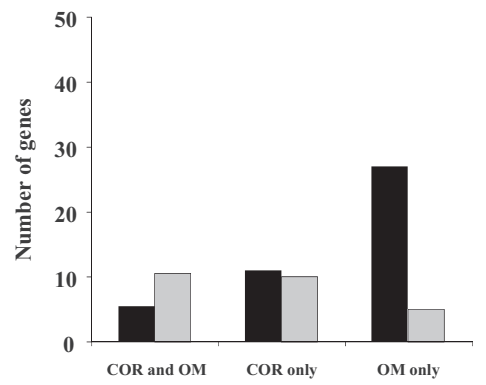

b)

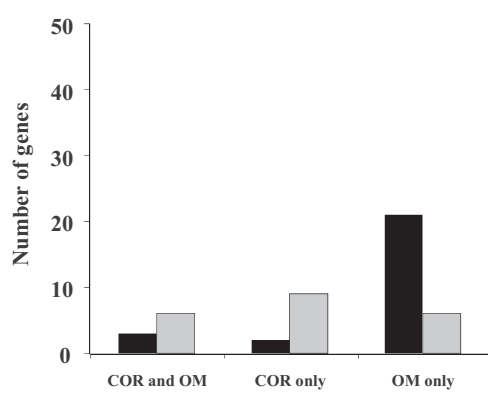

e)

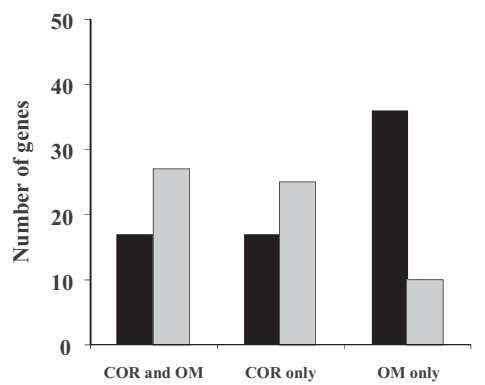

c)

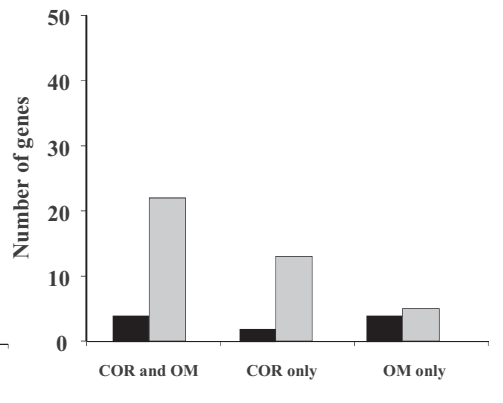

$\square:$ COR

: $\mathrm{OM}$

: Upregulation

: Downregulation

Fig. 1. The number of genes modulated by OTA in the COR and OM. The number of genes categorized into DNA damage and DNA damage repair, oxidative stress, the cell cycle, and apoptosis in the COR and OM (a). The number of genes up- or downregulated in the COR only, the OM only, and both the COR and OM in DNA damage and DNA damage repair (b), oxidative stress (c), the cell cycle (d), and apoptosis (e).

were extracted (Fig. 1a). A comparison of gene expression changes between the COR and OM in response to OTA was performed, and alterations in these genes are summarized according to molecular function.

\section{DNA damage- and DNA damage repair-related genes and their molecular functions}

Twenty-five genes, which were altered only in the OM, were differentially modulated by OTA treatment, and many of these genes were upregulated (Fig. $1 \mathrm{~b}$ and Table 1). In contrast, 11 genes were deregulated in the COR only, and many of these were downregulated (Fig. 1b and Supplementary Table 2). Nine genes were altered in both the COR and OM (Supplementary Table 3). In genes altered only in the OM, molecular functions were mainly classified as DNA double-strand break repair (DSBR), translesion synthesis (TLS), and $\mathrm{p} 53$-associated factors (Fig. 2a and Table 1). In order to confirm these data, representative genes altered only in the $\mathrm{OM}$ were analyzed by quantitative real-time PCR (Fig. 3). Most of the DNA DSBR related-genes, such as Chek1, Rad18, Bripl, and $B r c c 3$, were deregulated at both sites. However, the degree of change in gene expression was higher in the OM than in the COR. p53-associated factors (Phlda3 and Ccngl) were upregulated only in the OM, not in the COR. TLSrelated genes (Rev1 and Pcna) were not changed by OTA treatment at both sites.

\section{Oxidative stress-related genes and their molecular functions}

Nine genes in the OM (Fig. 1c and Table 1) and 15 genes in the COR (Fig. 1c and Supplementary Table 2) were differentially modulated by OTA treatment. In total, 26 genes were altered in both the COR and OM (Supplementary Table 3). In genes altered only in the OM, OTA-mediated functions included superoxide metabolic processes (Cyba, Cybb, and Sod1), glutathione metabolic processes (Gsta4, Gstt3, Gstk1, and Gpx8), and oxidative stress processes (Hmoxl) (Fig. $2 \mathrm{~b}$ and Table 1). Also, genes having the same function, such as Noxal, Gsta2, Gstm 1, and Gpxl were downregulated in the COR (Supplementary Table 2). 
Molecular mechanisms underlying OTA-induced genotoxicity

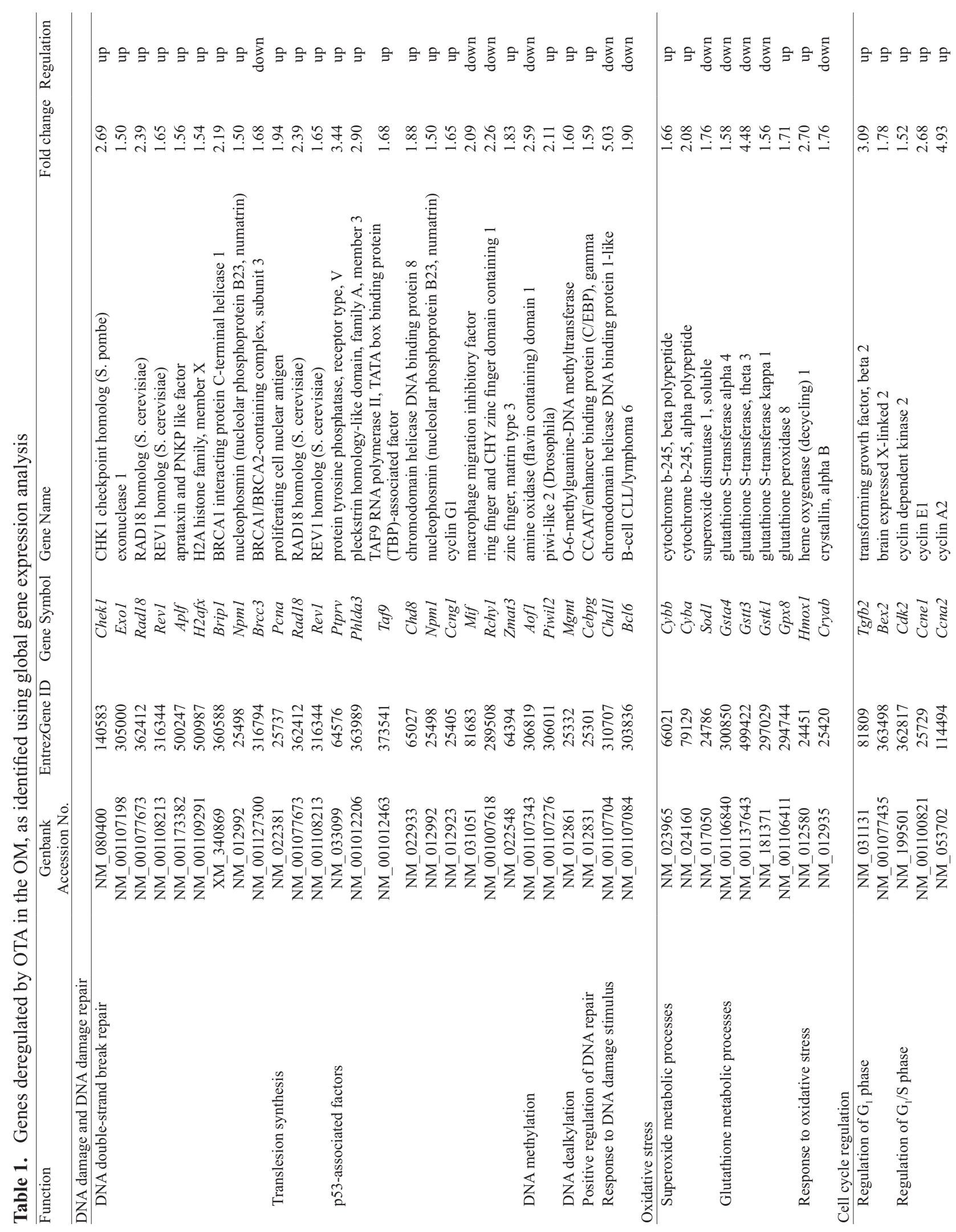


D. Hibi et al.

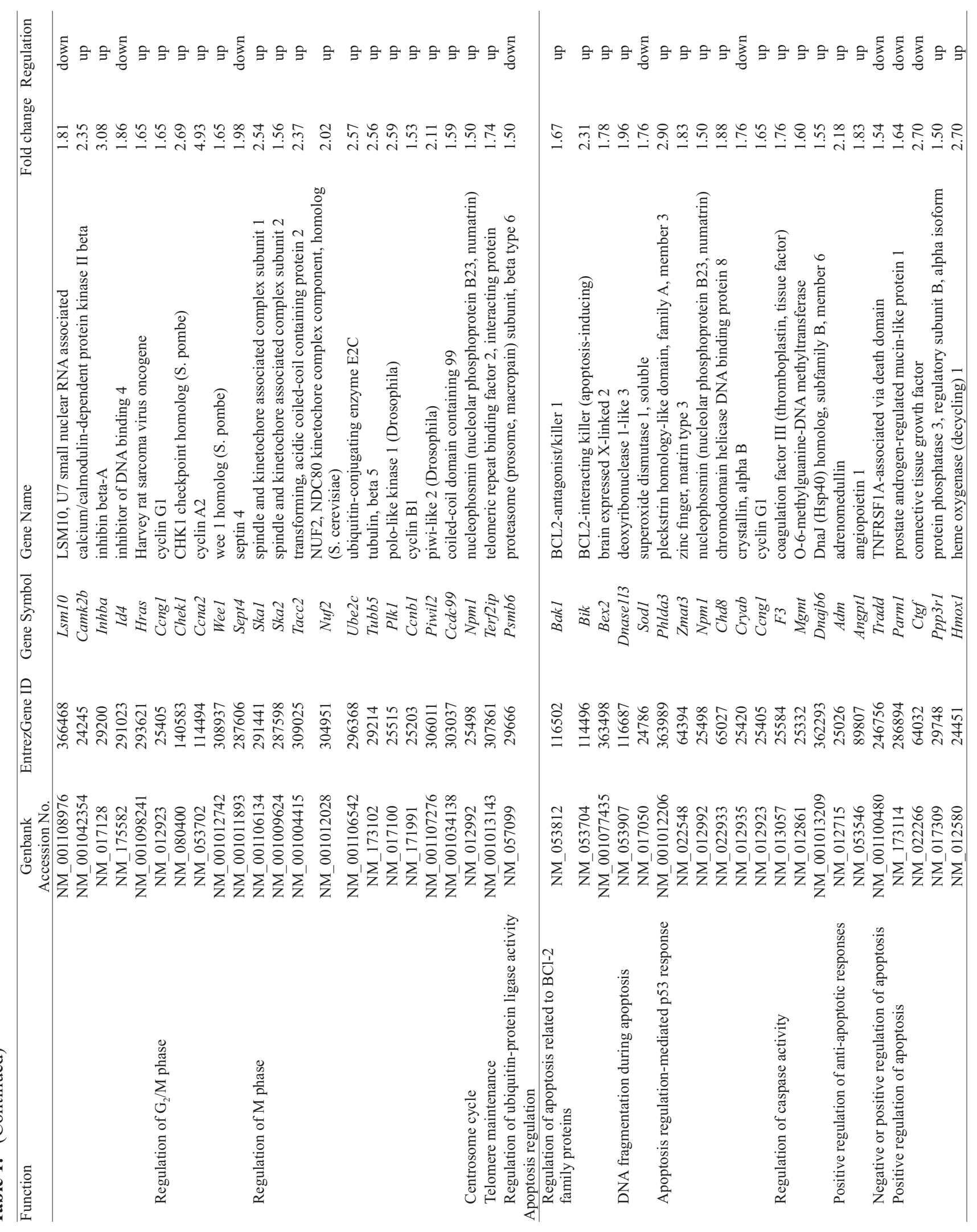

Vol. 38 No. 1 
Molecular mechanisms underlying OTA-induced genotoxicity

a)

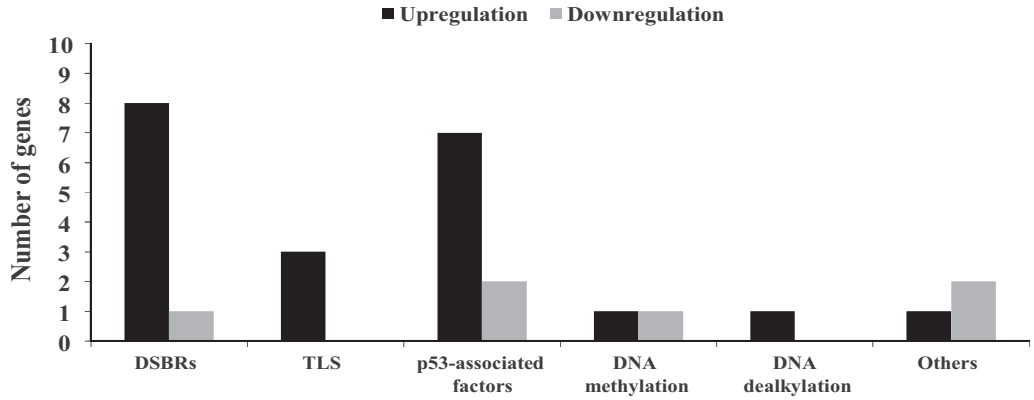

b)

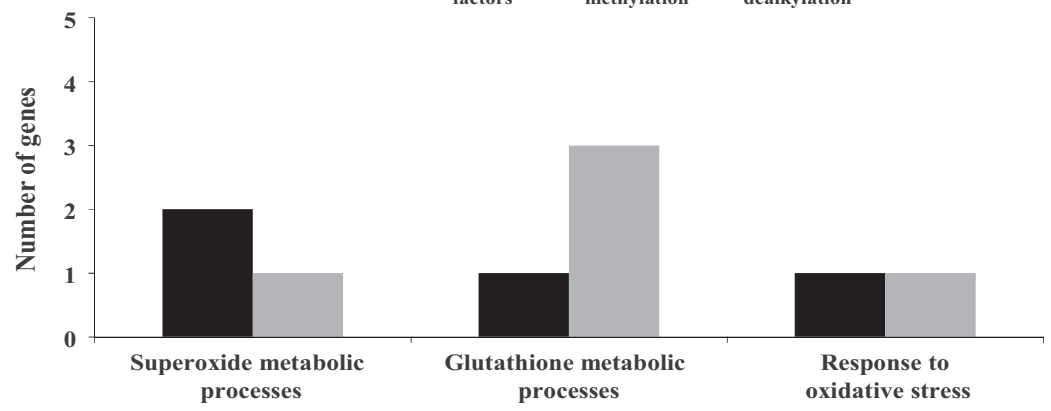

c)

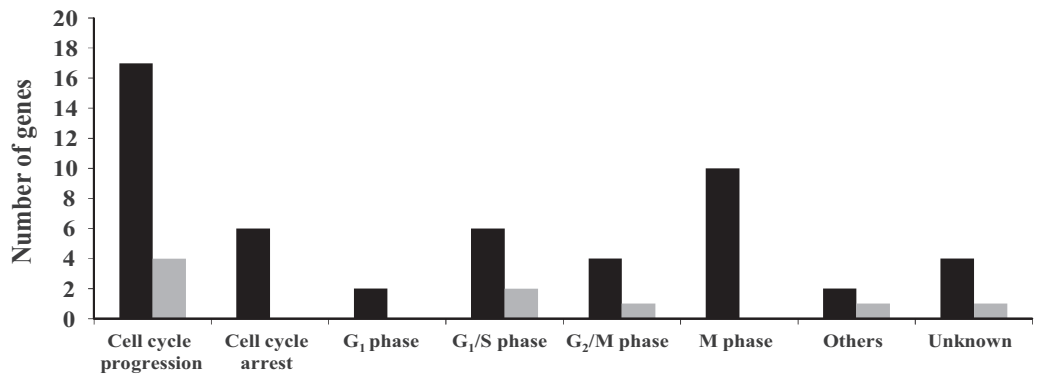

d)

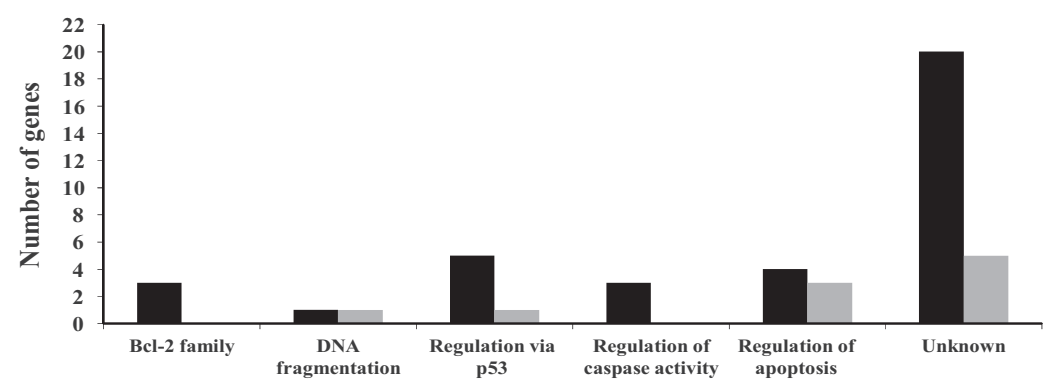

Fig. 2. Molecular functions of genes deregulated by OTA only in the OM. The number of genes up- or downregulated for each of the designated molecular functions: DNA damage and DNA damage repair (a), oxidative stress (b), the cell cycle (c), and apoptosis (d).

\section{Cell cycle regulation-related genes and their molecular functions}

In the OM, 34 genes were differentially modulated by OTA treatment, and many of these genes were upregulated (Fig. 1d and Table 1 and Supplementary Table 1). Fewer genes were altered in the COR than in the OM (Supplementary Table 2), and 14 genes were altered in both the COR and OM (Supplementary Table 3). The molecular functions of genes altered only in the OM included progression or arrest in each cell cycle phase, such as $\mathrm{G}_{1} / \mathrm{S}$ phase progression (Ccnel and Ccna2), $\mathrm{G}_{2} / \mathrm{M}$ or $\mathrm{M}$ phase progression (Ccna2 and $C c n b 1), \mathrm{G}_{2} / \mathrm{M}$ phase arrest (Ccng1, Chek1, and Wee1), and M phase mitotic process (Ska1, Ska2, Plk1, and so on; Fig. 2c and 


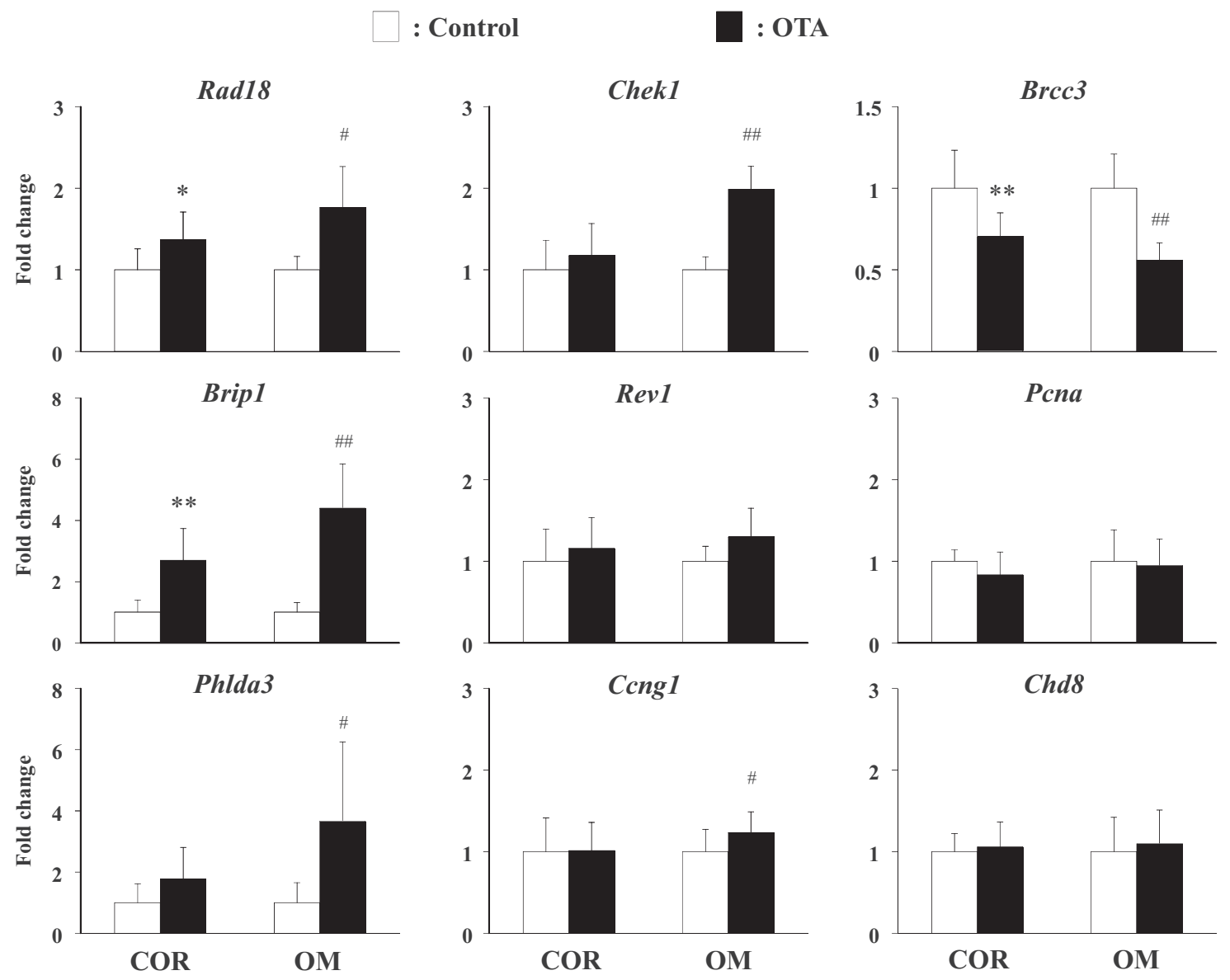

Fig. 3. Changes in mRNA levels of genes associated with DNA damage and DNA damage repair as measured by quantitative realtime RT-PCR. Data show changes in mRNA levels in the COR or OM. Values are means \pm SDs of data for 5 rats. ${ }^{*} P<0.05$ and ${ }^{* *} P<0.01$, significantly different from the COR in the control group. ${ }^{\sharp} P<0.05$ and ${ }^{\#} P<0.01$, significantly different from the $\mathrm{OM}$ in the control group.

Table 1 and Supplementary Table 1). Moreover, in quantitative real-time PCR analysis, Ccne1, Ccna2, Ccnb1, Ccng1, Chek1, and Weel were upregulated only in the OM, not in the COR (Figs. 3 and 4). These results suggested that OTA exerted abnormal regulatory effects on the cell cycle at the OTA target site.

\section{Apoptosis regulation-related genes and their molecular functions}

In the OM, 46 genes were differentially modulated by OTA treatment, and many of these genes were upregulated (Fig. 1e and Table 1 and Supplementary Table 1). In contrast, many of the 39 genes deregulated in the COR only were downregulated (Supplementary Table 2). Forty-two genes were altered in both the COR and OM
(Supplementary Table 3). In the OM, Bak1, Bik, and Bex2, categorized into the Bcl-2 family, and Phlda3, Zmat3, and $C h d 8$, involved in the apoptosis pathway and mediating the regulation of p53, were upregulated (Fig. 2d and Table 1 and Supplementary Table 1). In the COR, Bbc3 and $\mathrm{Bad}$, belonging to the Bcl-2 family, were upregulated, but no changes in the expression of genes involved in the apoptosis pathway and mediating the regulation of p53 were observed (Supplementary Table 2). In quantitative real-time PCR analysis, Bak1, Bik, and Phlda3 were upregulated in the OM, but not in the COR (Figs. 3 and 5).

\section{DISCUSSION}

Here, we have clarified the involvement of genotoxic 
Molecular mechanisms underlying OTA-induced genotoxicity

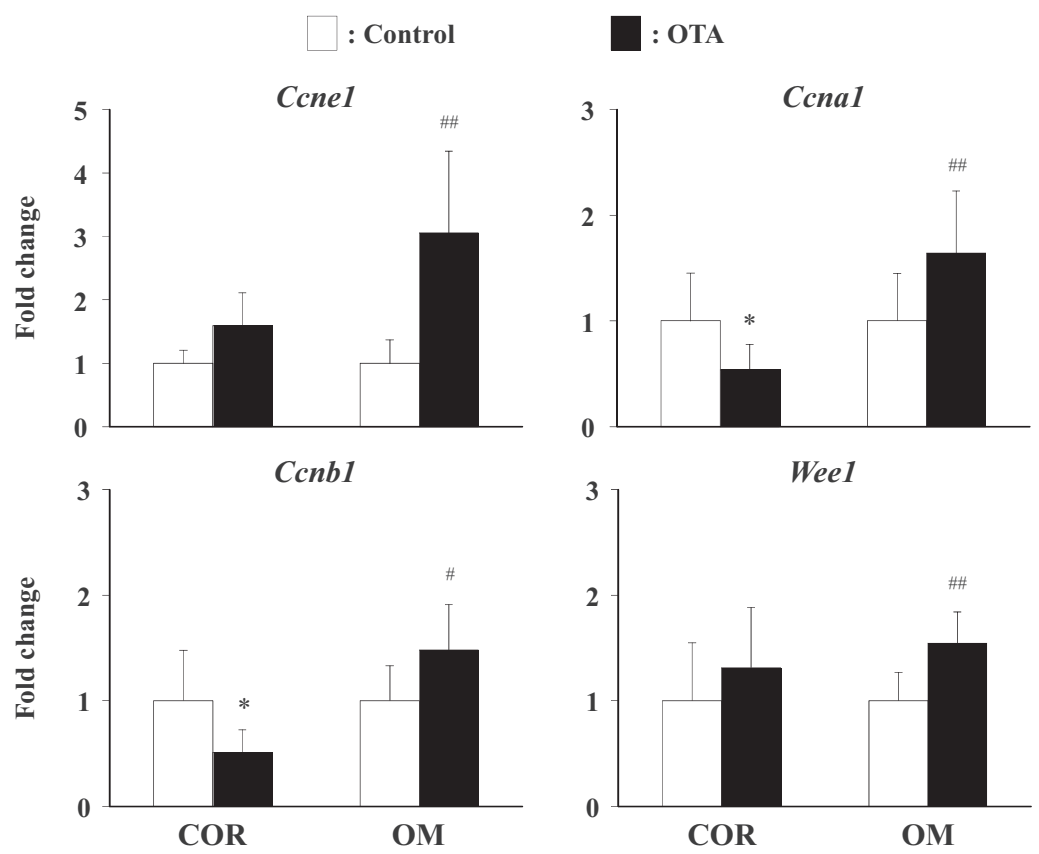

Fig. 4. Changes in mRNA levels of genes associated with the cell cycle as measured by quantitative real-time RT-PCR. Data show changes in mRNA levels in the COR or OM. Values are means \pm S.D.s of data for 5 rats. ${ }^{*} P<0.05$, significantly different from the COR in the control group. ${ }^{\#} P<0.05$ and ${ }^{\#} P<0.01$, significantly different from the OM in the control group.

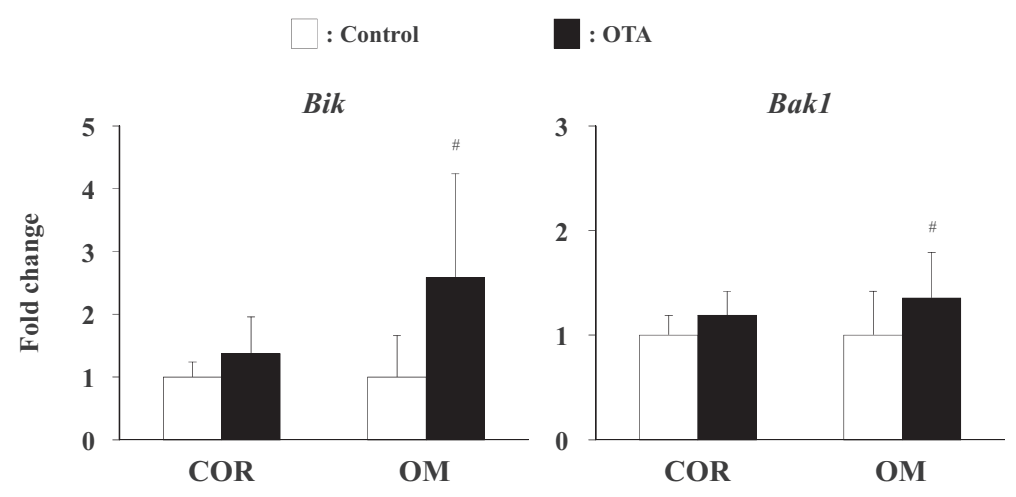

Fig. 5. Changes in mRNA levels of genes associated with apoptosis as measured by quantitative real-time RT-PCR. Data show changes in mRNA levels in the COR or OM. Values are means \pm S.D.s of data for 5 rats. ${ }^{\#} P<0.05$, significantly different from the $\mathrm{OM}$ in the control group.

mechanisms in OTA-induced renal carcinogenesis using reporter gene transgenic rats ( $g p t$ delta rats), in which deletion mutations may be predominantly induced by OTA. However, the mechanisms underlying OTA-induced mutation remain uncertain. In the present study, to identify promising candidate genes related to OTA-induced genotoxicity, we compared gene expression changes between the COR and OM in response to OTA exposure using additional samples from gpt delta rats under the same conditions used in our previous study (Hibi et al., 2011). Although a comprehensive gene analysis has already been performed (Marin-Kuan et al., 2006), here 


\section{Hibi et al.}

we have shown for the first time that genes associated with the DNA damage response were changed at the target site of OTA. In particular, genes encoding the several key regulators associated with DSBRs and p53-related factors were altered in response to OTA treatment in the OM. Moreover, some of genes related to DSBRs were also altered in the COR. However, more genes associated with DNA damage and DNA damage repair were altered in the OM than in the COR. These data suggested that DNA damage in the OM, a target site of OTA carcinogenicity, is more severe than in the COR.

DSBs are one of the most dangerous DNA damaging insults. Accordingly, protective systems, such as cell cycle arrest, induction of apoptosis, and DNA damage repair enzymatic networks, are active in vivo. DSBRs are generally conducted by 2 different pathways, nonhomologous end joining (NHEJ) and homologous recombination (HR) (Tsukamoto and Ikeda, 1998). The present results showed that the expression of several genes associated with DSBRs, such as Rad18 (Huang et al., 2009), Brip1 (Peng et al., 2006), Brcc3 (Chen et al., 2006), and Chek1 (Huang et al., 2008), all of which are involved in encoding HR repair enzymes for DSBs, fluctuated with OTA treatment. Also, changes in the mRNA expression of Phlda3 and Ccng1, which are regulated by p53, were found (Kawase et al., 2009; Okamoto and Beach, 1994). In light of a previous report demonstrating that OTA activated p53 in hepG2 cells (Bouaziz et al., 2008), these results may imply activation of p53 at the target site. Interestingly, p53 has been reported to be involved in HR repair for DSBs via interaction with Rad51 (Buchhop et al., 1997), BRCA1 (Ouchi et al., 1998), and replication protein A (RPA) (Dutta et al., 1993). In general, it has been thought that HR repair is an error-free repair process, inducing fewer gene mutations than other mechanisms. However, some reports have shown that that in the process of HR repair for DSBs, large deletion mutations, distinguishable from short deletions of 1 or $2 \mathrm{bp}$, were caused (Gebow et al., 2000; Bertrand et al., 1997). The formation of DNA modifications such as Benzo[a]pyrene-dihydrodiol epoxide and UV light-induced pyrimidine dimers enhance the replication stress at replication forks and thereby induce DSBs (Bi et al., 2005; Yajima et al., 2009). It has been reported that OTA resulted in DNA adducts formation (Mantle et al., 2010; Hadjeba-Medjdoub et al., 2012; Pfohl-Leszkowicz and Manderville, 2012) and the subsequent induction of AP sites (Cavin et al., 2007). Cleavage of AP sites by AP endonuclease has been considered to raise the possibility of the formation of single-strand breaks and then DSBs during the DNA replication (Caldecott, 2001). Additional function that OTA inhibited topoisomerase II activity (Cosimi et al., 2009) might contribute to the disorder of the normal DNA repair. Thus, although the mechanisms underlying OTA-induced DNA strand breaks including DSBs remain uncertain, positive results in the comet assay (Zeljezić et al., 2006; Klarić et al., 2010) support the hypothesis that OTA has the potential to cause DNA strand breaks.

Changes in the expression of genes associated with oxidative stress in the $\mathrm{OM}$ were not different from those in the COR in terms of the number of genes and the functions of individual genes. The present results found that various genes related to anti-oxidative enzymes, such as glutathione S-transferases, glutathione peroxidase, superoxide dismutase, heme oxygenase, and a component of NADPH oxidase, varied in response to OTA, consistent with a previous comprehensive analysis (Marin-Kuan et al., 2006). With respect to the possible pathways involved in OTA-induced oxidative stress, several mechanisms, including chelating ferric ions (Omar et al., 1990), ROS generation by redox cycling (Gillman et al., 1999; FaucetMarquis et al., 2006), and the reduction of anti-oxidative defenses resulting from the inactivation of Nrf2 transcription activity (Cavin et al., 2007), have been proposed. Thus, there is no doubt that OTA promotes ROS generation, and this was also supported by our current results. However, we previously showed that 8-OHdG levels (Hibi et al., 2011) and lipid peroxidation (data not shown) were not changed at the target site. In addition, the present data revealed no differences in the fluctuation of anti-oxidative-related genes between the COR and OM, strongly suggesting that OTA-induced oxidative stress does not contribute to its genotoxicity.

By comparison of global gene expression and RT-PCR analyses in the present study, the upregulation of various genes related to the cell cycle was found specifically in the OM; these included the following genes: Ccne1, associated with the cell cycle transition and/or progression to $\mathrm{G}_{1} / \mathrm{S}$ phase; Ccna2, associated with cell cycle progression to $\mathrm{S}$ or $\mathrm{G}_{2}$ phase; $C c n b 1$, associated with the cell cycle transition and/or progression to $\mathrm{G}_{2} / \mathrm{M}$ phase and inhibition of the cell cycle transition to $\mathrm{G}_{1} / \mathrm{M}$; Chek1, associated with the cell cycle checkpoint at $\mathrm{G}_{2} / \mathrm{M}$; and Wee1, associated with cell cycle arrest at $\mathrm{G}_{2} / \mathrm{M}$. Enhancement of cell proliferation at the S3 segment (Rached et al., 2007) and inhibition of cell cycle progression due to cell cycle arrest at $\mathrm{G}_{2} / \mathrm{M}$ phase (Cui et al., 2010) following exposure to OTA have already been reported; the present data were able to explain these phenomena by molecular changes. The disruption of normal mitosis through the expression of cyclin-dependent kinase inhibitors, topoisomerase II, and survivin may be responsible for the character- 
Molecular mechanisms underlying OTA-induced genotoxicity

istic observation of karyomegaly following OTA treatment (Adler et al., 2009; Rached et al., 2006). Also, the present data enable us to hypothesize possible molecular mechanisms involved in the development of karyomegaly observed at the OM, including upregulation of Chek1 acting at the $\mathrm{G}_{2} / \mathrm{M}$ checkpoint, Weel inducing $\mathrm{G}_{2} / \mathrm{M}$ arrest downstream of Chek1, and CcnA2, CcnB1, and CcnE1 mediating cell cycle progression. Together with a previous report demonstrating that Chk1 is activated by DNA strand breaks to induce polyploidy/aneuploidy during $\mathrm{G}_{2} / \mathrm{M}$ arrest (Zheng et al., 2011), the fact that HR repair mainly progresses during $\mathrm{G}_{2} / \mathrm{M}$ phase (Rothkamm et al., 2003) likely supports our proposal that predominant DNA lesion by OTA is DNA strand breaks.

The present data demonstrating changes in various p53-related genes, such as Bik and Bakl (belonging to the Bcl-2 family), Phlda3, and Ccngl may indicate activation of p53 in the OM, as mentioned above. Since OTA has been shown to induce apoptosis in proximal tubule cells of the S3 segment (Hibi et al., 2011; Rached et al., 2007), it is likely that p53-dependent apoptosis occurs in the OM. However, both the mitochondrial pathway, via the deregulation of Bcl-2 family proteins (Bouaziz et al., 2011; Assaf et al., 2004), and the p53-dependent pathway (Bouaziz et al., 2008) have been proposed. Bik, a protein identified in the present study, is activated by genotoxic stimuli through p53-dependent or -independent mechanisms, consequently leading to the induction of apoptosis through the mitochondrial pathway (Chinnadurai et al., 2008). Bak is also activated by p53, resulting in apoptosis through the mitochondrial pathway (Leu et al., 2004). Thus, while our data failed to clarify the role of p53 in OTA-induced apoptosis, it is likely that mitochondrialdependent pathways are involved.

In conclusion, the present data indicated that OTA induces strand breaks of target DNA including DSB and cell cycle progression, possibly leading to OTA-induced genotoxicity. Also, the present molecular analysis allows us to understand previously reported phenomena resulting from OTA treatment, such as observations of karyomegaly and apoptosis as consequences of OTA-induced DNA damage.

\section{ACKNOWLEDGMENTS}

We appreciate the expert technical assistance of Ms. Ayako Kaneko and Ms. Yoshimi Komatsu. This work was supported by a Grant-in-Aid for Technical Research on the Assessment of Food Effects on Health from the Food Safety Commission (\#0903) and a Grant-in-Aid for Cancer Research from the Ministry of Labor and Welfare of Japan.

\section{REFERENCES}

Adler, M., Müller, K., Rached, E., Dekant, W. and Mally, A. (2009): Modulation of key regulators of mitosis linked to chromosomal instability is an early event in ochratoxin A carcinogenicity. Carcinogenesis, 30, 711-719.

Assaf, H., Azouri, H. and Pallardy, M. (2004): Ochratoxin A induces apoptosis in human lymphocytes through down regulation of Bcl-xL. Toxicol. Sci., 79, 335-344.

Bendele, A.M., Carlton, W.W., Krogh, P. and Lillehoj, E.B. (1985): Ochratoxin A carcinogenesis in the (C57BL/6J X C3H)F 1 mouse. J. Natl. Cancer Inst., 75, 733-742.

Bertrand, P., Rouillard, D., Boulet, A., Levalois, C., Soussi, T. and Lopez, B.S. (1997): Increase of spontaneous intrachromosomal homologous recombination in mammalian cells expressing a mutant p53 protein. Oncogene, 14, 1117-1122.

Bi, X., Slater, D.M., Ohmori, H. and Vaziri, C. (2005): DNA polymerase kappa is specifically required for recovery from the benzo[a]pyrene-dihydrodiol epoxide (BPDE)-induced S-phase checkpoint. J. Biol. Chem., 280, 22343-22355.

Boorman, G.A., McDonald, M.R., Imoto, S. and Persing, R. (1992): Renal lesions induced by ochratoxin A exposure in the F344 rat. Toxicol. Pathol., 20, 236-245.

Bouaziz, C., Sharaf El Dein, O., El Golli, E., Abid-Essefi, S., Brenner, C., Lemaire, C. and Bacha, H. (2008): Different apoptotic pathways induced by zearalenone, $\mathrm{T}-2$ toxin and ochratoxin A in human hepatoma cells. Toxicology, 254, 19-28.

Bouaziz, C., Sharaf El Dein, O., Martel, C., El Golli, E., Abid-Essefi, S., Brenner, C., Lemaire, C. and Bacha, H. (2011): Molecular events involved in ochratoxin A induced mitochondrial pathway of apoptosis, modulation by Bcl-2 family members. Environ. Toxicol., 26, 579-590.

Buchhop, S., Gibson, M.K., Wang, X.W., Wagner, P., Stürzbecher, H.W. and Harris, C.C. (1997): Interaction of p53 with the human Rad51 protein. Nucleic. Acids. Res., 25, 3868-3874.

Caldecott, K.W. (2001): Mammalian DNA single-strand break repair: an X-ra(y)ted affair. Bioessays, 23, 447-455.

Cavin, C., Delatour, T., Marin-Kuan, M., Holzhäuser, D., Higgins, L., Bezençon, C., Guignard, G., Junod, S., RichozPayot, J., Gremaud, E., Hayes, J.D., Nestler, S., Mantle, P. and Schilter, B. (2007). Reduction in antioxidant defenses may contribute to ochratoxin A toxicity and carcinogenicity. Toxicol. Sci., 96, 30-39.

Chen, X., Arciero, C.A., Wang, C., Broccoli, D. and Godwin, A.K. (2006): BRCC36 is essential for ionizing radiation-induced BRCA1 phosphorylation and nuclear foci formation. Cancer Res., 66, 5039-5046.

Chinnadurai, G., Vijayalingam, S. and Rashmi, R. (2008): BIK, the founding member of the $\mathrm{BH} 3$-only family proteins: mechanisms of cell death and role in cancer and pathogenic processes. Oncogene, 27, S20-29.

Cosimi, S., Orta, L., Mateos, S. and Cortés, F. (2009): The mycotoxin ochratoxin A inhibits DNA topoisomerase II and induces polyploidy in cultured CHO cells. Toxicol. In Vitro, 23, 11101115.

Cui, J., Xing, L., Li, Z., Wu, S., Wang, J., Liu, J., Wang, J., Yan, X. and Zhang, X. (2010): Ochratoxin A induces G(2) phase arrest in human gastric epithelium GES-1 cells in vitro. Toxicol. Lett., 193, 152-158.

Dahlmann, A., Dantzler, W.H., Silbernagl, S. and Gekle, M. (1998): Detailed mapping of ochratoxin A reabsorption along the rat 


\section{Hibi et al.}

nephron in vivo: the nephrotoxin can be reabsorbed in all nephron segments by different mechanisms. J. Pharmacol. Exp. Ther. 286, 157-162.

Dutta, A., Ruppert, J.M., Aster, J.C. and Winchester, E. (1993): Inhibition of DNA replication factor RPA by p53. Nature, 365, 79-82.

European Food Safety Authority (EFSA) (2006): Opinion of the Scientific Panel on Contaminants in the Food Chain on a Request from the Commission Related to Ochratoxin A in Food. EFSA J., 365, 1-56.

Faucet-Marquis, V., Pont, F., Størmer, F.C., Rizk, T., Castegnaro, M. and Pfohl-Leszkowicz, A. (2006): Evidence of a new dechlorinated ochratoxin A derivative formed in opossum kidney cell cultures after pretreatment by modulators of glutathione pathways: correlation with DNA-adduct formation. Mol. Nutr. Food. Res., 50, 530-542.

Gebow, D., Miselis, N. and Liber, H.L. (2000): Homologous and nonhomologous recombination resulting in deletion: effects of p53 status, microhomology, and repetitive DNA length and orientation. Mol. Cell. Biol., 20, 4028-4035.

Gillman, I.G., Clark, T.N. and Manderville, R.A. (1999): Oxidation of ochratoxin A by an Fe-porphyrin system: model for enzymatic activation and DNA cleavage. Chem. Res. Toxicol., 12, 10661076.

Hadjeba-Medjdoub, K., Tozlovanu, M., Pfohl-Leszkowicz, A., Frenette, C., Paugh, R.J. and Manderville, R.A. (2012): Structure-activity relationships imply different mechanisms of action for ochratoxin A-mediated cytotoxicity and genotoxicity. Chem. Res. Toxicol., 25, 181-190.

Hibi, D., Suzuki, Y., Ishii, Y., Jin, M., Watanabe, M., Sugita-Konishi, Y., Yanai, T., Nohmi, T., Nishikawa, A. and Umemura, T. (2011): Site-specific in vivo mutagenicity in the kidney of gpt delta rats given a carcinogenic dose of ochratoxin A. Toxicol. Sci., 122, 406-414.

Huang, J., Huen, M.S., Kim, H., Leung, C.C., Glover, J.N., Yu, X. and Chen, J. (2009): RAD18 transmits DNA damage signaling to elicit homologous recombination repair. Nat. Cell. Biol., 11, 592-603.

Huang, M., Miao, Z.H., Zhu, H., Cai, Y.J., Lu, W. and Ding, J. (2008): Chk1 and Chk2 are differentially involved in homologous recombination repair and cell cycle arrest in response to DNA double-strand breaks induced by camptothecins. Mol. Cancer Ther., 7, 1440-1449.

Joint FAO/WHO Expert Committee on Food Additives (JECFA). (2001): Ochratoxin A. JECFA Food Additive Series, 47, 1-132.

JECFA (2008): Safety evaluation of certain food additives and contaminants. WHO Food Additives Series, 59, 357-429.

Kamp, H.G., Eisenbrand, G., Janzowski, C., Kiossev, J., Latendresse, J.R., Schlatter, J. and Turesky, R.J. (2005): Ochratoxin A induces oxidative DNA damage in liver and kidney after oral dosing to rats. Mol. Nutr. Food Res., 49, 1160-1167.

Kawase, T., Ohki, R., Shibata, T., Tsutsumi, S., Kamimura, N., Inazawa, J., Ohta, T., Ichikawa, H., Aburatani, H., Tashiro, F. and Taya, Y. (2009): PH domain-only protein PHLDA3 is a p53regulated repressor of Akt. Cell, 136, 535-550.

Klarić, M.S., Darabos, D., Rozgaj, R., Kasuba, V. and Pepeljnjak, S. (2010): Beauvericin and ochratoxin A genotoxicity evaluated using the alkaline comet assay: single and combined genotoxic action. Arch. Toxicol., 84, 641-650.

Kumata, K., Amano, R., Ichinoe, M. and Uchiyama, S. (1980): Culture conditions and purification method for large-scale production of ochratoxins by Aspergillus ochraceus, Shokuhin
Eiseigaku Zasshi, 21, 171-176.

Leu, J.I., Dumont, P., Hafey, M., Murphy, M.E. and George, D.L. (2004): Mitochondrial p53 activates Bak and causes disruption of a Bak-Mcll complex. Nat. Cell Biol., 6, 443-450.

Mantle, P., Kulinskaya, E. and Nestler, S. (2005): Renal tumourigenesis in male rats in response to chronic dietary ochratoxin A. Food Addit. Contam., 22, S58-64.

Mantle, P.G., Faucet-Marquis, V., Manderville, R.A., Squillaci, B. and Pfohl-Leszkowicz, A. (2010): Structures of covalent adducts between DNA and ochratoxin A: a new factor in debate about genotoxicity and human risk assessment. Chem. Res. Toxicol., 23, 89-98.

Marin-Kuan, M., Nestler, S., Verguet, C., Bezençon, C., Piguet, D., Mansourian, R., Holzwarth, J., Grigorov, M., Delatour, T., Mantle, P., Cavin, C. and Schilter, B. (2006): A toxicogenomics approach to identify new plausible epigenetic mechanisms of ochratoxin A carcinogenicity in rat. Toxicol. Sci., 89, 120-134.

National Toxicology Program (NTP) (1989): Toxicology and Carcinogenesis Studies of Ochratoxin A (CAS No. 303-47-9) in F344/N Rats (Gavage Studies). Natl. Toxicol. Program Tech. Rep. Ser., 358, 1-142.

Nohmi, T. and Masumura, K. (2005): Molecular nature of intrachromosomal deletions and base substitutions induced by environmental mutagens. Environ. Mol. Mutagen., 45, 150-161.

Nohmi, T., Suzuki, T. and Masumura, K. (2000): Recent advances in the protocols of transgenic mouse mutation assays. Mutat. Res., 455, 191-215.

Okamoto, K. and Beach, D. (1994): Cyclin G is a transcriptional target of the p53 tumor suppressor protein. EMBO J. 13, 48164822 .

Omar, R.F., Hasinoff, B.B., Mejilla, F. and Rahimtula, A.D. (1990): Mechanism of ochratoxin A stimulated lipid peroxidation. Biochem. Pharmacol., 40, 1183-1191.

Ouchi, T., Monteiro, A.N., August, A., Aaronson, S.A. and Hanafusa, H. (1998): BRCA1 regulates p53-dependent gene expression, Proc. Natl. Acad. Sci. USA, 95, 2302-2306.

Peng, M., Litman, R., Jin, Z., Fong, G. and Cantor, S.B. (2006): BACH1 is a DNA repair protein supporting BRCA1 damage response. Oncogene, 25, 2245-2253.

Pfohl-Leszkowicz, A. and Manderville, R.A. (2007): Ochratoxin A: an overview on toxicity and carcinogenicity in animals and humans. Mol. Nutr. Food Res., 51, 61-99.

Pfohl-Leszkowicz, A. and Manderville, R.A. (2012): An update on direct genotoxicity as a molecular mechanism of ochratoxin A carcinogenicity. Chem. Res. Toxicol., 25, 252-262.

Rached, E., Hard, G.C., Blumbach, K., Weber, K., Draheim, R., Lutz, W.K., Ozden, S., Steger, U., Dekant, W. and Mally, A. (2007): Ochratoxin A: 13-week oral toxicity and cell proliferation in male F344/N rats. Toxicol. Sci., 97, 288-298.

Rached, E., Pfeiffer, E., Dekant, W. and Mally, A. (2006): Ochratoxin A: apoptosis and aberrant exit from mitosis due to perturbation of microtubule dynamics? Toxicol. Sci., 92, 78-86.

Rothkamm, K., Krüger, I., Thompson, L.H. and Löbrich, M. (2003): Pathways of DNA double-strand break repair during the mammalian cell cycle. Mol. Cell Biol., 23, 5706-5715.

Schaaf, G.J., Nijmeijer, S.M., Maas, R.F., Roestenberg, P., de Groene, E.M. and Fink-Gremmels, J. (2002): The role of oxidative stress in the ochratoxin A-mediated toxicity in proximal tubular cells. Biochim. Biophys. Acta., 1588, 149-158.

Tsukamoto, Y. and Ikeda, H. (1998): Double-strand break repair mediated by DNA end-joining. Genes Cells, 3, 135-144.

Yajima, H., Lee, K.J., Zhang, S., Kobayashi, J. and Chen, B.P. 
Molecular mechanisms underlying OTA-induced genotoxicity

(2009): DNA double-strand break formation upon UV-induced replication stress activates ATM and DNA-PKcs kinases. J. Mol. Biol., 385, 800-810.

Zeljezić, D., Domijan, A.M. and Peraica, M. (2006): DNA damage by ochratoxin $\mathrm{A}$ in rat kidney assessed by the alkaline comet assay. Braz. J. Med. Biol. Res., 39, 1563-1568.
Zheng, L., Dai, H., Hegde, M.L., Zhou, M., Guo, Z., Wu, X., Wu, J., Su, L., Zhong, X., Mitra, S., Huang, Q., Kernstine, K.H., Pfeifer, G.P. and Shen, B. (2011): Fen1 mutations that specifically disrupt its interaction with PCNA cause aneuploidy-associated cancer. Cell Res., 21, 1052-1067. 